\title{
Análisis de la atenuación de emisiones conducidas en sistemas complejos
}

\author{
Julio Guillermo Zola $^{\# 1}$, Pablo Marino Belcaguy ${ }^{\# 2}$, Federico Gabriel D’Angiolo ${ }^{\# 3}$ \\ \# Laboratorio de Circuitos Electrónicos, Dto. de Electrónica, Facultad de Ingeniería, Universidad de Buenos Aires \\ Paseo Colón 850 (1063), CABA, Argentina \\ 1 jzolaefi.uba.ar \\ 2 pablo.marino.belcaguyegmail.com \\ 3 federicogd2009@gmail.com \\ Recibido: 31/10/16; Aceptado: 10/07/17
}

\begin{abstract}
The analysis and attenuation of electromagnetic interference (EMI) in complex electronic systems is an important issue to take into account in the design of these systems, due to malfunctions that may be caused by such interferences. In this paper the particular design of a filter to reduce spurious currents or conducted emissions from different DC to DC converters that are part of a complex system, is described. This design includes a method to help to reduce conducted emissions from these converters. While design criteria are oriented to a particular system, this study can be applied generically to any system where it is necessary to evaluate EMI.
\end{abstract}

Resumen- El análisis y atenuación de la interferencia electromagnética (EMI) en sistemas electrónicos complejos resulta un aspecto de importancia a tener en cuenta en su diseño, debido al mal funcionamiento que pueden provocar estas interferencias en dichos sistemas. En el presente trabajo se describe el diseño particular de un filtro para atenuar las corrientes espurias o emisiones conducidas provenientes de distintos conversores de continua a continua que forman parte de un sistema complejo. Incluye este diseño un método para ayudar a reducir las emisiones conducidas desde dichos conversores. Si bien, los criterios de diseño usados están orientados para la electrónica de un sistema particular, este estudio puede aplicarse en forma genérica a cualquier sistema donde sea necesario evaluar EMI.

\section{INTRODUCCIÓN}

En muchos sistemas donde existen dispositivos semiconductores que conmutan, se producen corrientes indeseadas las cuales se propagan por todo el sistema a través del conexionado. Según la topología de dicho sistema, se define la forma en la cual dichas corrientes terminan contribuyendo, como por ejemplo el caso en que todos los subsistemas se encuentren conectados a una línea principal de alimentación o bus principal [1]. En el presente trabajo, estas corrientes espurias se generan en convertidores o conversores de continua a continua (DC-DC). Estas corrientes espurias en los DC-DC, que ya han sido modelizadas en trabajos anteriores [2], se propagan a través del cableado de alimentación hacia distintos bloques circuitales que componen un equipamiento complejo. El efecto de estas corrientes o emisiones conducidas (CE) puede traer como consecuencia la degradación del funcionamiento de cada bloque, provocando así el deterioro general del sistema. La inmunidad de cada bloque en general se evalúa mediante ensayos de susceptibilidad conducida (CS) o inmunidad conducida [3,4].

Por ejemplo, si algún bloque que se conecta a esta línea de alimentación contiene circuitos digitales, estos podrían producir errores en las codificaciones digitales, provocando así su mal funcionamiento. Otro ejemplo se da en la emisión y recepción de imágenes, es decir en un sistema que se encuentra constantemente transmitiendo imágenes a través de señales. Si dichas señales digitales son afectadas por las $\mathrm{CE}$, puede ocurrir que las imágenes recibidas sean confusas o no tengan el suficiente detalle requerido.

Para poder evaluar la influencia de estas corrientes espurias, se debe analizar la interferencia electromagnética sobre el sistema (EMI), que es la emisión de energía electromagnética que degrada o perjudica la calidad de una señal $[2,5,6]$. Por otro lado, debe evaluarse asimismo la compatibilidad electromagnética (EMC), es decir la habilidad de un sistema de no causar EMI a otros equipos, así como de ser insensible a las emisiones que puedan causar otros sistemas [7]. Tanto las CE como la CS son evaluadas mediante ensayos dedicados y existen normas bien conocidas al respecto $[8,9,10,17]$.

\section{PROPAGACIÓN DE LAS EMISIONES CONDUCIDAS}

Se puede decir que existe por un lado un equipo "culpable" encargado de emitir las interferencias y por otro un equipo receptor o "victima", que recibe las interferencias, tal como se muestra en el esquema de la Fig. 1 [11].

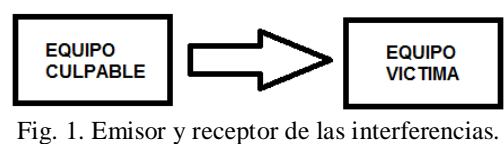

En este caso se toma como equipo emisor a un DC-DC, pero en general los circuitos electrónicos de potencia son los equipos "culpables", ya que para mejorar su rendimiento trabajan en conmutación. Los dispositivos que producen esta conmutación (diodos, transistores, etc.) conectan y desconectan niveles elevados de corrientes y tensiones. Estas conexiones y desconexiones producen señales eléctricas no deseadas que afectan a otros equipos electrónicos y que dan origen a las EMI.

En la Fig. 2 se muestran las variaciones temporales de corrientes y tensiones que tienen mayor contribución a las CE en un DC-DC básico de tipo flyback [12]. Al conmutar el transistor, se producen corrientes que tienen un alto contenido armónico. La corriente en el transformador evoluciona linealmente con la tensión aplicada en sus terminales, por lo tanto esta tensión también contribuirá al espectro agregando componentes armónicas. 
Estas señales espurias pueden transmitirse de dos formas: por radiación (transmisión por el espacio) o por conducción (CE). O sea, en este último caso, las señales se transmiten a través de los conductores [5]. Un modo de transmisión domina frente al otro dependiendo de la frecuencia y las longitudes de onda (más específicamente de la longitud eléctrica del medio en el que se propaga, es decir de la relación entre la longitud de onda y la longitud física) [2,6]. Las CE están comprendidas en un rango de frecuencias hasta $30 \mathrm{MHz}$ o $50 \mathrm{MHz}$ aproximadamente, mientras que las EMI por radiación se encuentran por encima hasta $1 \mathrm{GHz}$, estando sus valores normalizados hasta los $40 \mathrm{GHz}$ $[5,8,9,10]$.
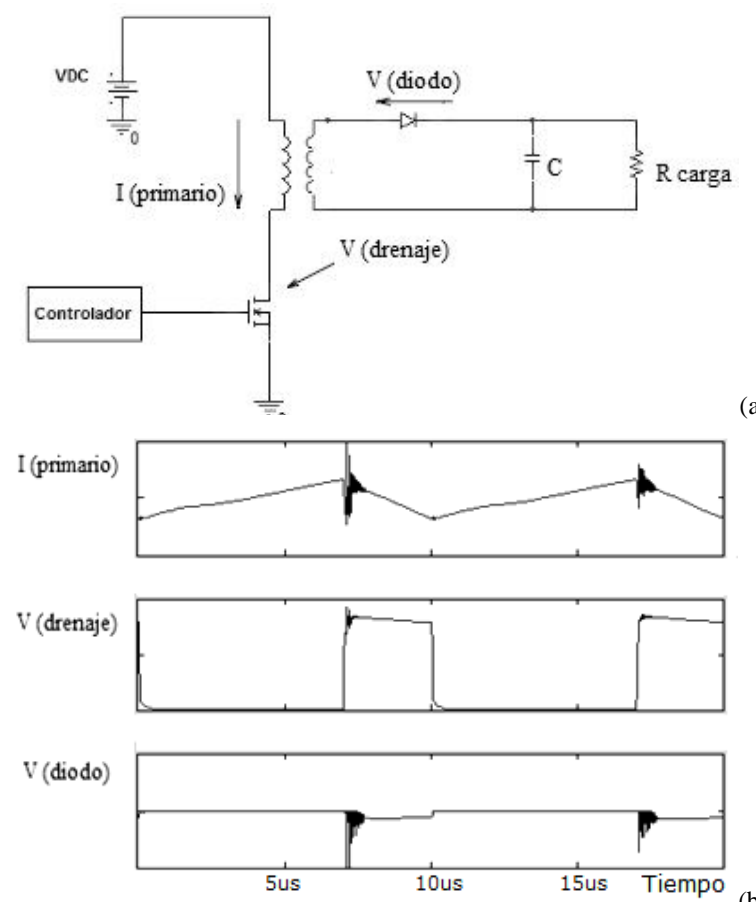

Fig. 2. (a) Circuito básico de un DC-DC tipo flyback. (b) Corriente en el primario del transformador, tensión en el drenaje y tensión sobre el diodo.

Las CE, al propagarse por medio de cables o conductores, se pueden transmitir de dos formas: en modo diferencial (MD) o en modo común (MC) [5,13]. En el primer caso las señales viajan en sentido opuesto entre dos líneas que llegan a un mismo bloque, tal como muestra la Fig. 3a. En el segundo caso, las señales se propagan en el mismo sentido con respecto a tierra o chasis $(O V)$, tal como se muestra en la Fig. 3b.

Cuando el DC-DC es parte de un sistema más complejo, una forma de atenuar estas corrientes espurias, es conectar un filtro $L C$ entre el equipo emisor y el receptor de las CE. Se propone un filtro $L C$ y no $R C$, dado que los niveles de corriente por el bus, provocarían pérdidas de potencia.

La necesidad del agregado de este filtro en forma externa y no interno en el DC-DC, se puede deber a causas diversas tales como: peso del equipo, dimensiones, costos o simplemente porque se utiliza un DC-DC comercial sin posibilidad de realizarle modificaciones a su diseño.

En la Fig. 3c se muestra un sistema genérico, donde se justifica la ubicación del filtro $L C$ : una fuente eléctrica alimenta a través de una línea o bus de alimentación diferentes bloques (que representan otros componentes conectados al mismo bus de potencia, en la tensión de alimentación primaria del DC-DC) y en particular a un DCDC que alimenta a su vez a otros bloques funcionales " $\mathrm{X}$ ", conectados al secundario del DC-DC. Se asume que el capacitor de salida del DC-DC atenuará las CE de alta frecuencia hacia el bloque " $X$ ", por lo que el sentido de las CE a través del sistema será el indicado por las flechas. Es decir, el estudio se centra en las CE del lado primario del DC-DC. En la Fig. 3d se observa una forma de medición de las $\mathrm{CE}$ a la entrada y salida del filtro.

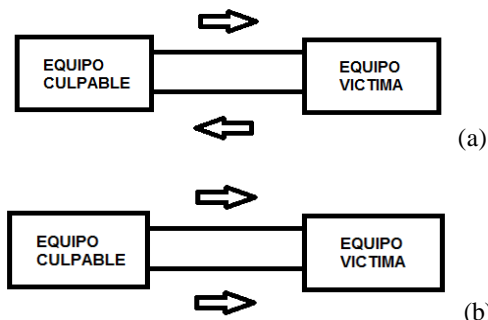

(b)

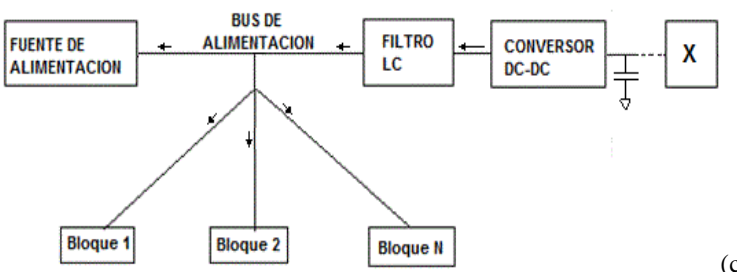

(c)

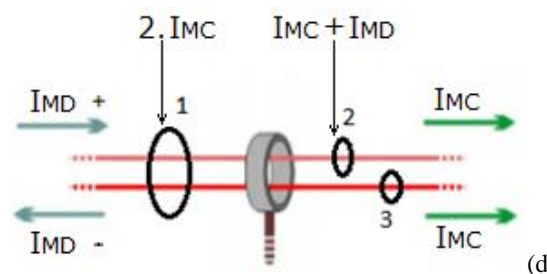

Fig. 3. (a) Propagación de CE en MD. (b) Propagación de CE en MC. (c) Sistema genérico mostrando los sentidos de circulación de CE y la ubicación del filtro LC. (d) Método de medición de las CE a la entrada y salida del filtro, utilizando puntas de corriente.

\section{DISEÑO DEL FILTRO}

Para diseñar el filtro deben evaluarse los perfiles o máscaras propuestos para el sistema particular, que son gráficas que indican los máximos niveles permitidos de CE dentro de un rango de frecuencias determinado. Con estas máscaras se puede deducir la atenuación que debe tener el filtro. Debe cumplir además con algunas condiciones impuestas por el sistema y por su circuitería interna (por ejemplo, valores límite de inductancia, capacitancia, tamaño, etc.). Por ejemplo, en un sistema complejo tal como la electrónica de un avión, resulta de suma importancia el peso y tamaño del filtro a diseñar, así como el tipo de componentes que se utilicen ya que deben resistir condiciones extremas, tales como bajas temperaturas y fuertes vibraciones, por citar algunos ejemplos. Asimismo, el valor de inductancia del filtro a conectar a la entrada de un DC-DC podría provocar un funcionamiento inestable en el conversor si su valor superase un máximo determinado.

Para obtener la característica de transferencia que debe cumplir el filtro, hay que considerar el perfil de CE que se tiene del DC-DC y el perfil al que se desea llegar o que 
impone el sistema. En la Fig. 4a se muestra la máscara de $\mathrm{CE}$ en MD provenientes del DC-DC, es decir los niveles máximos emitidos por el DC-DC en MD. La máscara de CE es función de las características de la unidad de potencia dada por sus características constructivas y de diseño. Por otro lado, la Fig. 4b muestran los límites máximos aceptados en el receptor o equipo víctima, también denominado máscara.

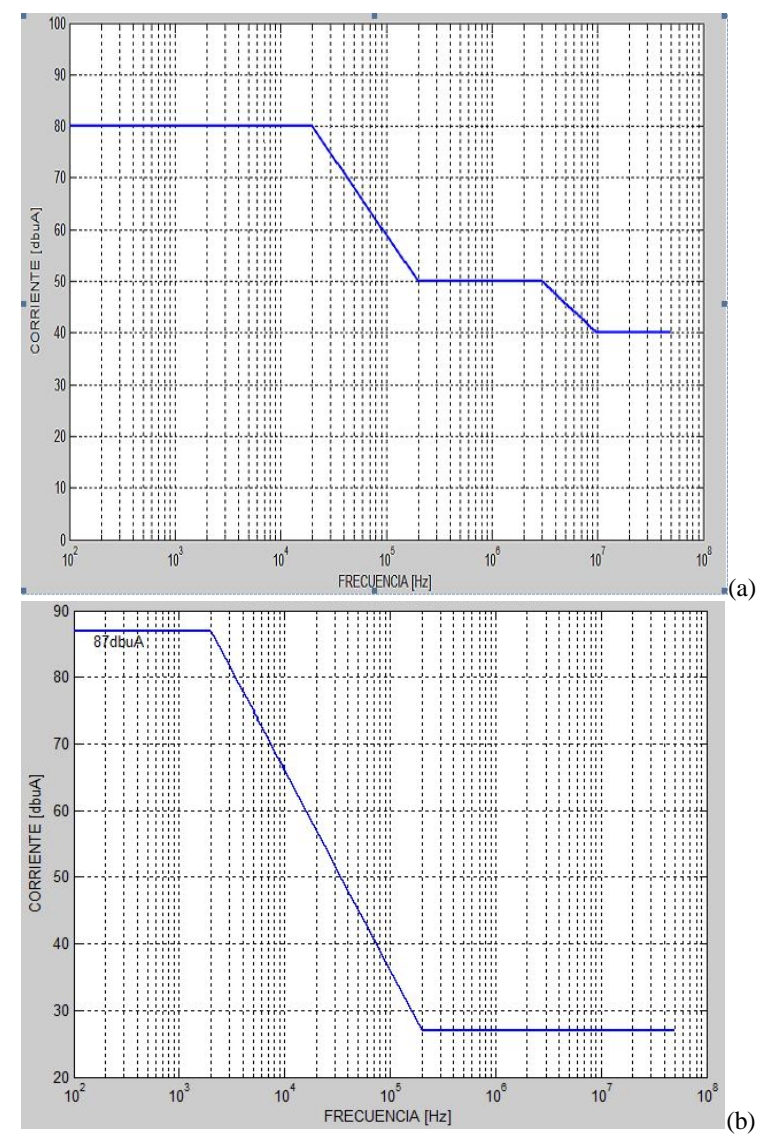

Fig. 4. (a) Máscara de CE en MD provenientes de un conversor DC-DC, (b) Máscara de $\mathrm{CE}$ en $\mathrm{MD}$ requerida a nivel del equipo receptor.

En general, para analizar las EMI se emplean como unidades de medida $d b u A$ y $d b u V$ [14], como se ve en (1).

$$
d b \mu A=20 \log \left(\frac{I_{1}}{1 \mu A}\right) ; d b \mu V=20 \log \left(\frac{V_{1}}{1 \mu V}\right)
$$

Tomemos el siguiente ejemplo: si diez DC-DC destinados a alimentar a un conjunto de bloques funcionales se conectan a un bus de alimentación, la máscara de la Fig. $4 \mathrm{~b}$ representará los niveles máximos que pueden recibir dichos bloques conectados al bus cuando los diez DC-DC se encuentran en funcionamiento.

Conociendo entonces los niveles de CE para un DC-DC, se estiman los niveles de CE que emiten diez DC-DC. La contribución de CE de los diez DC-DC se hará sumando los respectivos valores eficaces de sus corrientes espurias (suma cuadrática), tal como se muestra en (2). No se considerará el peor caso, dado por la suma aritmética directa de las corrientes, ya que resulta improbable que estén en fase.

$$
I_{\text {total }}=\sqrt{I_{1}^{2}+I_{2}^{2}+\ldots+I_{10}^{2}}=\sqrt{10 I_{1}^{2}}=\sqrt{10} \cdot I_{1}
$$

donde $I_{i}$ es la corriente que entrega el DC-DC i-ésimo.

Teniendo en cuenta que 20.log $(10)^{1 / 2}=10 \mathrm{db}$, la máscara para diez conversores DC-DC será como la indicada en la Fig. 4a pero desplazada $10 \mathrm{db}$, tal como se muestra en la Fig. 5.

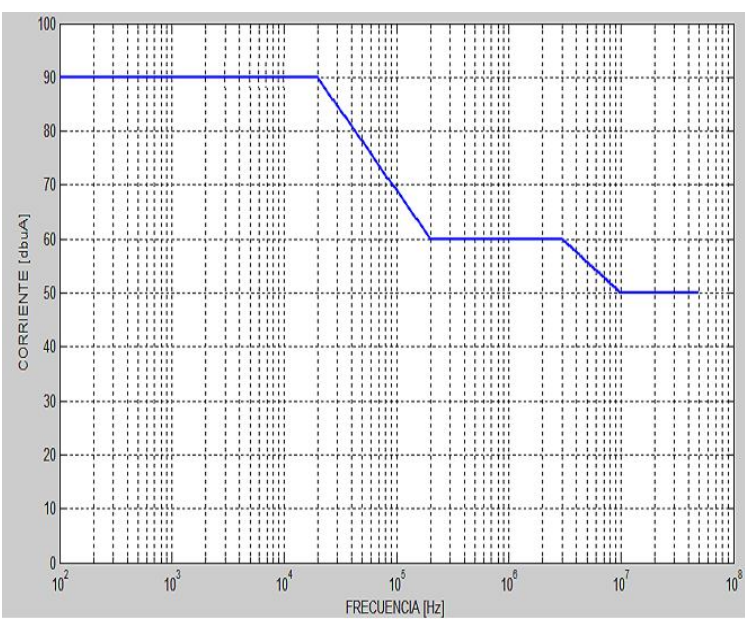

Fig. 5. Máscara de CE provenientes de diez conversores DC-DC.

Con este último dato y la máscara de CE a nivel del equipo víctima, se puede aplicar la expresión (3) y obtener la atenuación del filtro requerida, que se muestra en la Fig. 6.

$$
A t t(d b)=I_{0}(d b \mu A)-I_{\text {total }}(d b \mu A)
$$
filtro.

donde $I_{0}$ es la corriente debida a las $\mathrm{CE}$ atenuadas por el

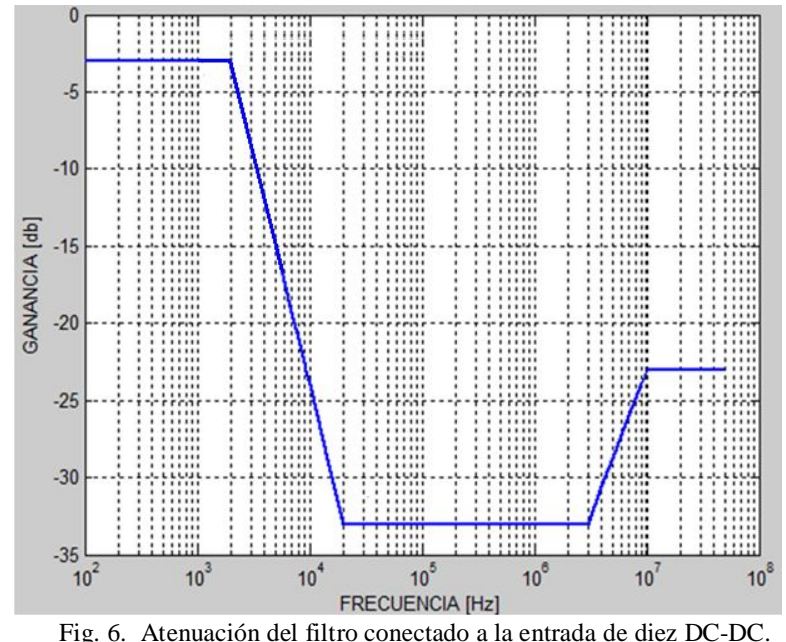

En la Fig. 6 se muestra la atenuación teórica del filtro para el ejemplo dado. El hecho de que por encima de los $20 \mathrm{kHz}$ ya no atenúe de igual modo, no es necesario tenerlo en cuenta en la implementación del filtro. Un filtro pasa bajos con el valor de transferencia adecuado a $20 \mathrm{kHz}$, 
atenuará todas las componentes de frecuencias superiores a $2 \mathrm{kHz}$.

En la Fig. 7 se muestra un filtro genérico que cumple con gráficas similares a la Fig. 6 (salvo el cambio de pendiente en la atenuación, como se explicó en el párrafo anterior) tanto para MD como para MC. El bloque formado por $L d$ y $C d$ será el encargado de atenuar las CE de MD. Su forma simétrica resulta indicada para la atenuación de estas CE. Por otro lado, para atenuar las CE de MC, se agrega un par de inductores acoplados $L c$ colocados en la rama serie del filtro. Los bornes homólogos de estos inductores se encuentran del mismo lado y dado que las corrientes de MC se propagan en el mismo sentido, producen campos magnéticos con la misma orientación que al enfrentarse se cancelan. De esta forma, los inductores acoplados presentan una alta impedancia al flujo de corrientes de MC, pero una baja impedancia para MD, es decir no intervienen en el filtrado del MD.

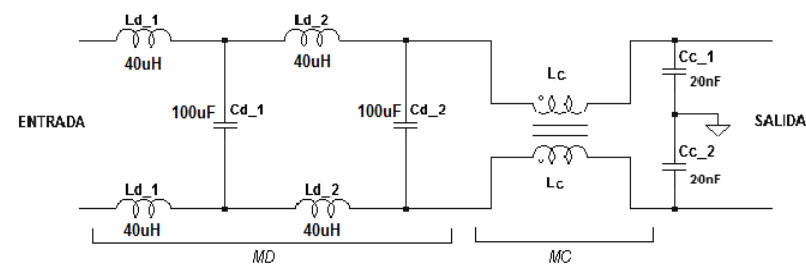

Fig. 7. Circuito esquemático del filtro (el esquema y los valores son sólo de referencia).

Las capacitancias $C c$ son las parásitas respecto de tierra que presenta el sistema, cuyos valores dependerán del sistema en particular, pero podrían estimarse en los $n F$. En $\mathrm{MD}$ no tienen influencia pero sí en $\mathrm{MC}$, ya que las $\mathrm{CE}$ retornan a tierra a través de ellas. En particular, para $\mathrm{MC}$ el filtro puede reducirse al indicado en la Fig. 8 [15].

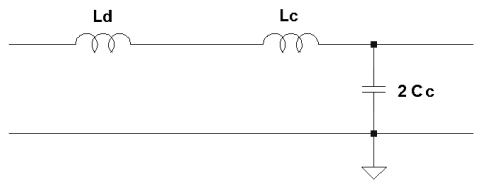

Fig. 8. Equivalente del filtro en MC.

Por lo tanto, el proceso de diseño del filtro sería, obtener el bloque que atenúa el MD y luego, conocidos $L d$ y $C d$, obtener $L c$ a partir del circuito de la Fig. 8. Teniendo en cuenta los valores de la Fig. 7, Lc sería de $200 \mathrm{mH}$, aproximadamente.

\section{SOLUCIÓN ALTERNATIVA PARA REDUCIR EL MODO COMÚN}

En ocasiones, en sistemas complejos, la limitación en los valores de los componentes a utilizar en el filtro, por las razones dadas en cuanto al peso, espacio, etc., podrían limitar su eficacia. Especialmente en nuestro ejemplo, el valor de $L c$ obtenido para la adecuada reducción de las CE de MC implica construir inductores acoplados de tamaño y peso que pueden ser excesivos para los requerimientos del sistema bajo estudio.

No serviría aumentar la capacitancia en paralelo $C c$ para compensar una disminución de $L c$, dado que ello traería como consecuencia una reducción de la aislación eléctrica del sistema. Por ello, se analiza una solución alternativa, la cual consiste en mantener el par de inductores $L c$, pero con valores mucho menores al calculado y agregar una red circuital en el DC-DC que ayude a reducir las CE de MC que emite.

En los DC-DC la capacidad distribuida en el transformador favorece la conducción de corrientes en MC. En un transformador ideal, las corrientes de MC no tendrían lugar dado que no producen una diferencia de potencial en el primario. En un transformador real, las capacitancias parásitas existentes entre primario y secundario debido a la separación entre los devanados así como al tipo y espesor del dieléctrico que los separa, pueden producir un camino posible para las corrientes de MC [16]. Estas capacitancias se las puede representar en forma distribuida en ambas ramas, tal como se muestra en la Fig. 9. Esta distribución no tiene por qué ser uniforme, por ejemplo por irregularidades en el espesor del dieléctrico. De esta manera, habrá un desbalance en el flujo de corriente en cada rama, o sea aparecerán corrientes de MD debidas a señales de entrada de MC.

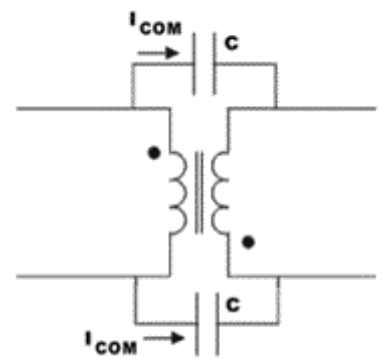

Fig. 9. Efecto de las corrientes de MC sobre un transformador real.

Como la distribución de las capacitancias parásitas dependerá de la construcción del transformador, para solucionar este desequilibrio entre las corrientes de MC, se plantea una modificación como la indicada en el ejemplo de la Fig. 10, donde se muestra un modelo de señal de un DCDC similar al indicado en la Fig. 2a, con el agregado de un capacitor $C f$.

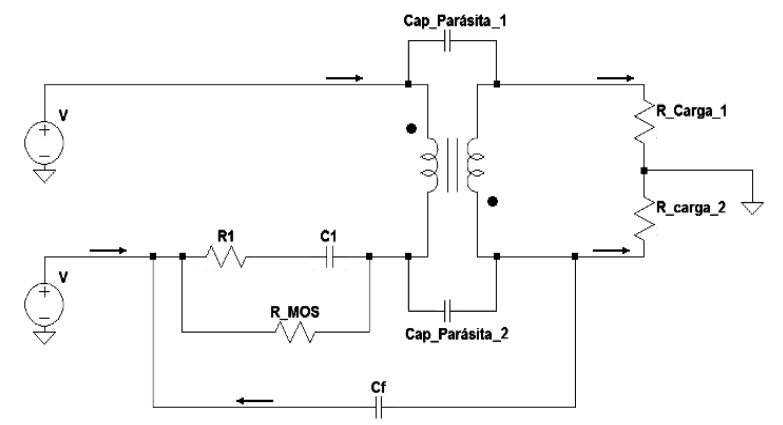

Fig. 10. Circuito de señal del DC-DC con el agregado de $C f$ para equilibrar las corrientes de MC.

Las capacitancias Cap_parásita son las parásitas del transformador, $R \_M O S$ es la resistencia que presenta el transistor MOS en la conmutación y el filtro formado por Cl y Rl forma parte del circuito de conmutación. Los valores de las cargas equivalentes para la señal común, $R \_$carga, representan al diodo de rectificación del conversor en conjunto con su respectiva carga. El capacitor agregado $C f$ muestrea la tensión del secundario e inyecta su corriente 
al circuito del primario. El propósito es el de producir un equilibrio entre ambas ramas del circuito. Si la corriente en el secundario por la rama inferior fuese mayor respecto de la superior, la tensión respecto de tierra sobre $R \_$carga_2 aumenta. En ese caso, se inyecta corriente a la entrada por $C f$. Esto produce una disminución en la tensión del terminal homólogo del primario, bajando consecuentemente la tensión en el terminal homólogo del secundario. Para que esta realimentación sea efectiva, el valor de $C f$ no debería ser muy cercano al de los componentes parásitos ni muy grande como para reducir seriamente la aislación del transformador.

\section{Conclusiones}

El análisis de las CE y su reducción resulta ser un punto importante a tener en cuenta al implementar sistemas electrónicos complejos. Se debe buscar, especialmente al tratar de reducir las $\mathrm{CE}$ de $\mathrm{MC}$, en buscar soluciones alternativas que permitan el uso de componentes de menor tamaño y peso, sin afectar la aislación eléctrica.

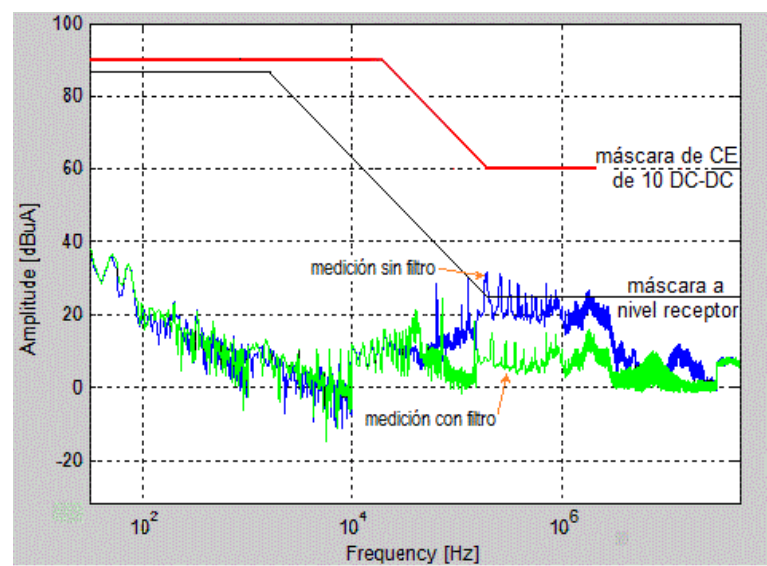

Fig. 11. Medición de CE en MC con filtro conectado.

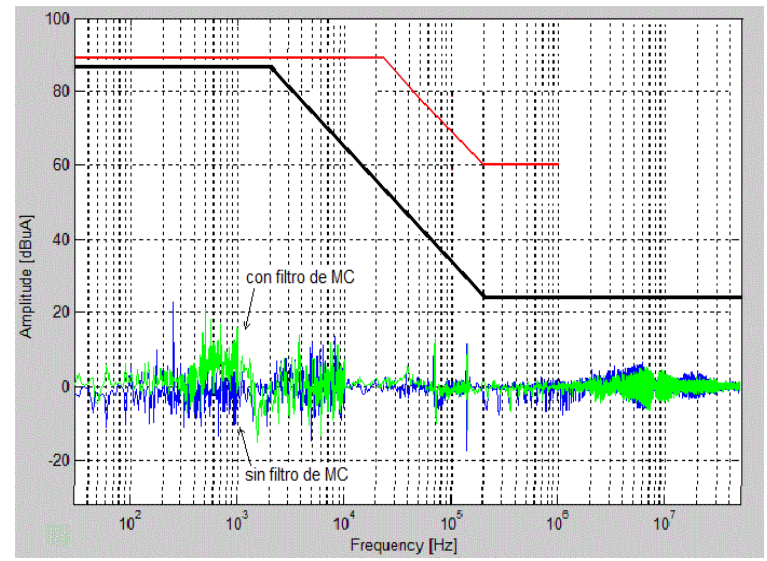

Fig. 12. Medición de CE para MD, agregando y quitando las etapas que atenúan el MC.
La solución propuesta en este trabajo lo logra en principio, tal como puede observarse en la Fig. 11, donde se muestra el resultado de mediciones sobre el sistema ante excitaciones de señales espurias de MC. La atenuación alcanza los $10 \mathrm{db}$ aproximadamente, por la acción del filtro conformado por el par de inductores $L c$ y el capacitor $C f$ en el DC-DC.

Asimismo, como se muestra en la Fig. 12, el bloque MD del filtro de la Fig. 7 resulta muy eficiente para atenuar la incidencia de señales espurias de MD, las cuales se ubican muy por debajo de la máscara correspondiente. Puede notarse además que la inclusión de la etapa MC, junto con el capacitor $C f$ de realimentación entre primario y secundario del DC-DC, no afecta a la atenuación de las señales de MD.

\section{REFERENCIAS}

[1] Space Engineering. Electromagnetic Compatibility Handbook, ECSS-E-HB-20-07a, September 5th, 2012.

[2] V. W. Greb, "Controlling Conducted Emissions from DC-DC Converters", Ball Aerospace Boulder Co., 1995.

[3] D.J. Atkins and P. Bisognin, "Prediction and Measurement of Spacecraft Conducted Safety Margin", 10th International Conference on Electromagnetic Compatibility, London, UK, 1997.

[4] C. Ruiz and E. Gonzalez, "EMI/RFI por Sistemas de Respaldo Eléctrico en Telecomunicaciones", Posgrado de Ingeniería Eléctrica UC. Sistemas de Respaldo de Energía, 2004.

[5] L. Ferrer i Arnau, "Introducción a la compatibilidad electromagnética", UPC Departament d'Enginyeria Electrònica, Barcelona, Spain, 2012.

[6] Clayton Paul, Introduction to Electromagnetic Compatibility, 2nd Edition, Wiley Interscience, 2006.

[7] H. W. Ott, Electromagnetic Compatibility Engineering, John Wiley \& Sons., 2009.

[8] Electromagnetic Emission and Susceptibility Requirements for the Control of Electromagnetic Interference, MIL-STD-461, 2013.

[9] Measurements of Electromagnetic Interference Characteristics, MILSTD-462, 1999.

[10] Electromagnetic Compatibility Requirements for Space System, MIL-STD-1541, 1987.

[11] D. F. Knurek, "Reducing EMI In Switch Mode Power Supply", 10th International Telecommunications Energy Conference (INTELEC'88), San Diego, USA, 1988.

[12] K.R. Aravind Britto, R. Dhanasekaran, R. Vimala, B. Saranya, "EMI Analysis and Evaluation of an Improved Flyback Converter", International Conference on Computer Communication and Informatics (ICCCI), Coimbatore, India, 2012.

[13] H. K. Patel, "Flyback Power Supply EMI Signature and Supression Techniques", Joint International Conference on Power System Technology and IEEE Power India Conference, India, 2008.

[14] L. A. Mallete and R. Adams, "An Introduction to EMI/EMC Test Requirements for Space Applications", IEEE Aerospace Conference. Proceedings, 1999.

[15] P. Wang, C. Tao, J. Zhang, "Research and Design of a Common Mode Hybrid EMI Filter for Switch-mode Power Supply", 3rd International Conference on Power Electronics Systems and Applications, Hong Kong, 2009.

[16] Understanding Common Mode Noise, GO19A, Pulse a Technitrol Company, 4/1999.

[17] Space Engineering. Electromagnetic Compatibility, ECSS-E-ST-2007C, 2008. 